\title{
Crystal structure, morphology and formation mechanism of a novel polymorph of lead dioxide, $\gamma-\mathrm{PbO}_{2}$
}

\section{Hiba Kabbara, Jaafar Ghanbaja, Abdelkrim Redjaïmia and Thierry Belmonte}

J. Appl. Cryst. (2019). 52, 304-311

\section{IUCr Journals CRYSTALLOGRAPHY JOURNALS ONLINE}

Copyright (C) International Union of Crystallography

Author(s) of this article may load this reprint on their own web site or institutional repository provided that this cover page is retained. Republication of this article or its storage in electronic databases other than as specified above is not permitted without prior permission in writing from the IUCr.

For further information see http://journals.iucr.org/services/authorrights.html 


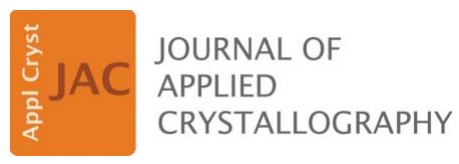

ISSN 1600-5767

Received 25 June 2018

Accepted 21 January 2019

Edited by G. Renaud, CEA-Grenoble DSM/ INAC/SP2M/NRS, Grenoble, France

\# Present address: CNRS, Laboratoire des Sciences des Procédés et des Matériaux, UPR3407, Villetaneuse F-93430, France.

Keywords: discharge in liquid nitrogen; lead dioxide nanosheets; hexagonal crystal system; morphology; formation mechanisms.

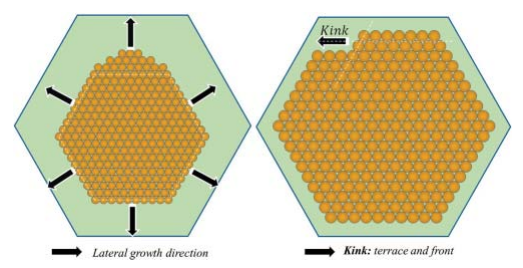

(C) 2019 International Union of Crystallography

\section{Crystal structure, morphology and formation mechanism of a novel polymorph of lead dioxide, $\gamma-\mathrm{PbO}_{2}$}

\author{
Hiba Kabbara, ${ }^{a} \ddagger$ Jaafar Ghanbaja, ${ }^{\mathrm{a}}$ Abdelkrim Redjaïmia ${ }^{\mathrm{a}}$ and Thierry Belmonte ${ }^{\mathrm{a}, \mathrm{b} *}$

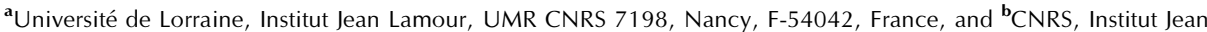 \\ Lamour, UMR CNRS 7198, Nancy, F-54042, France. *Correspondence e-mail: thierry.belmonte@univ-lorraine.fr
}

Nanosecond-pulsed spark discharge between two lead electrodes in liquid nitrogen has been used to synthesize hexagonal lead nanosheets. These original nanostructures are collected on a substrate located under the electrodes. After the full evaporation of the liquid nitrogen, the nanosheets are oxidized in air and transform into a lead dioxide. The resulting hexagonal sheets have typical widths of around $1 \mu \mathrm{m}$ and typical thicknesses of around $10 \mathrm{~nm}$. Investigations by energy dispersive spectroscopy microanalysis, transmission electron microscopy, high-angle annular dark-field scanning transmission electron microscopy and electron microdiffraction were performed in order to identify the crystal structure in which these hexagonal nanosheets crystallize. An analysis of the chemical composition pointed to a stoichiometric lead dioxide, $\mathrm{PbO}_{2}$. This $\mathrm{PbO}_{2}$ lead dioxide crystallizes in the hexagonal system $(a=0.912 \mathrm{~nm}$ and $c=1.265 \mathrm{~nm})$ and belongs to the space group $P 6 / \mathrm{m} 2 / \mathrm{m} 2 / \mathrm{m}$. On the basis of group theory (symmetry analysis), the nanosheets develop a hexagonal-prismatic shape in liquid nitrogen, assumed to be an isotropic medium. From the energetic point of view, this shape, dictated by the $6 / \mathrm{m} 2 / \mathrm{m} 2 / \mathrm{m}$ point group, corresponds to an absolute extremum, an indicator of the stability of this lead dioxide. A mechanism similar to that of the ledge mechanism explaining the formation of thin plates in a metallic matrix has been adapted and proposed for the formation of the lead nanosheets in the liquid nitrogen. When the liquid nitrogen is removed, the lead nanosheet is oxidized, leading to a lead dioxide, inheriting the nanosheet morphology. As far as the authors are aware, this is the first time that such a lead dioxide has been synthesized by spark discharge in liquid nitrogen followed by oxidation in air. The crystallographic structure is determined and the morphology is explained. A mechanism for the development of the lead nanosheets and their oxidation is proposed. This hexagonal phase, designated $\gamma-\mathrm{PbO}_{2}$, is thought to be the third polymorph after the $\alpha-\mathrm{PbO}_{2}$ and $\beta-\mathrm{PbO}_{2}$ phases of lead dioxide, the former being orthorhombic and the latter being tetragonal.

\section{Introduction}

Creating high-voltage discharges in liquid nitrogen is a method developed nearly 15 years ago to produce original nanostructures (Sano et al., 2004; Charinpanitkul et al., 2009; Hamdan et al., 2018) by electrode erosion in non-equilibrium conditions (Hamdan et al., 2013; Belmonte et al., 2014). This process is known to exhibit one of the highest production yields of nano-objects by a physical method.

This simple method has attracted interest for mass production at low cost. In particular, original lead nanostructures have been recently synthesized by plasma in liquid nitrogen. These nanostructures, which can be used for various applications, are also attractive thanks to their superconductivity properties (Strongin et al., 1970; Haviland et al., 1989), their magnetic properties (Aramini et al., 1996) etc. This 


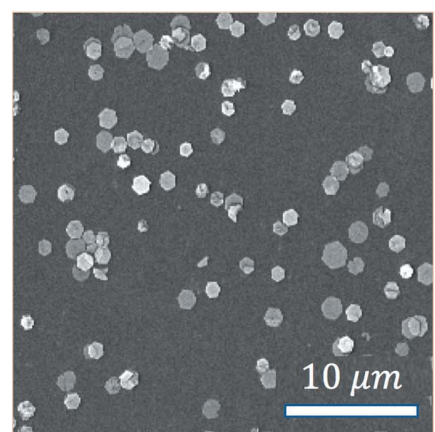

(a)

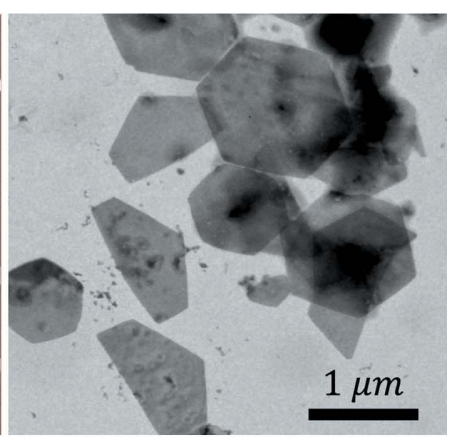

(b)
Figure 1

(a) Scanning electron microscopy and (b) bright-field TEM images showing lead dioxide particles with hexagonal shape, deposited on silicon (a) and TEM carbon grid (b) substrates.

method initially produces hexagonal nanosheets. However, the synthesis of lead nanosheets is limited by a specific erosion process that produces lead sticks beyond about 2000 discharges rather than hexagonal nanosheets [see the detailed explanation given by Hamdan et al. (2018)]. X-ray diffraction being not relevant, we have used energy dispersive spectroscopy (EDS), transmission electron microscopy (TEM), highangle annular dark-field scanning transmission electron microscopy (HAADF-STEM) and electron diffraction to identify the crystal structure of lead nanosheets obtained by this process, in order to explain the lead dioxide morphology inherited from the hexagonal lead nanosheet particles.

A mechanism is proposed to explain the development of the lead nanosheets in the liquid nitrogen, followed by their oxidation in air, giving lead dioxide nanosheets.

\section{Experimental setup}

The experimental setup for the synthesis of lead oxide nanosheets is presented in detail in the paper recently published by Hamdan et al. (2018). Briefly, by pulsed discharges in liquid nitrogen between two wires made of lead and used as electrodes in a pin-to-pin configuration, hexagonal nanosheets were obtained (Fig. 1).

Carbon and holey carbon grids were used as substrates to collect the products of the electrode erosion process, in order to characterize them, among others, by electron microscopy. After full evaporation of the liquid nitrogen, the lead nanosheets are exposed to air and oxidized in an uncontrolled manner. TEM investigations were carried out using a JEMARM200F cold field emission gun (FEG) and a Philips CM200 device operating at 200 and $120 \mathrm{kV}$, respectively. The chemical composition was determined by EDS via the JEM-ARM $200 \mathrm{~F}$ cold FEG.

Finally, the CrystalMaker software (http://crystalmaker. com) was used to generate simulated electron diffraction patterns. These simulated patterns were useful to eliminate irrelevant structures among 230 down to the final possibility. Note that there is no JCPDS file of lead oxide that could be used to index the diffraction patterns.

\section{Characterization of the lead dioxide nanosheets}

\subsection{Chemical analysis}

After evaporation, the lead nanosheets exposed to air are oxidized and form a lead oxide. The chemical composition of this lead oxide was investigated by EDS. Fig. 2(a) shows a typical EDS spectrum displaying lead and oxygen, the basic elements entering into the chemical composition of the oxide nanosheet. The quantification of these two constituent elements indicates a homogenous lead dioxide, $\mathrm{PbO}_{2}$. However, a few particles do not respect this stoichiometric homogeneity across the whole particle [Figs. 2(b) and 2(c)].

A profile of the radial distribution of lead and oxygen through a particular nanosheet [Fig. 2(b)] is shown in Fig. 2(c). Across the profile, the chemical composition is homogenous, except in the central zone. The lead oxide continuously changes from lead dioxide $\left(\mathrm{PbO}_{2}\right)$ to lead monoxide $(\mathrm{PbO})$. The oxygen/lead atomic ratio goes from $2\left(\mathrm{PbO}_{2}\right)$ to $1(\mathrm{PbO})$. The central area, whose diameter is around $0.5 \mu \mathrm{m}$, is then

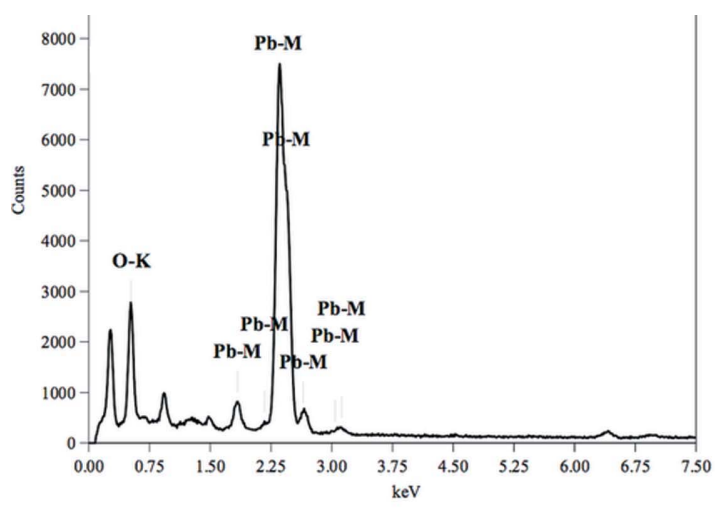

(a)

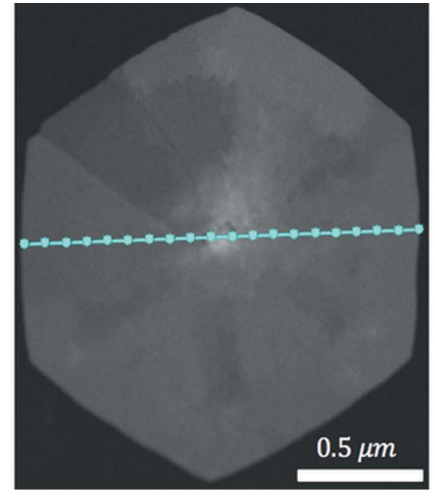

(b)

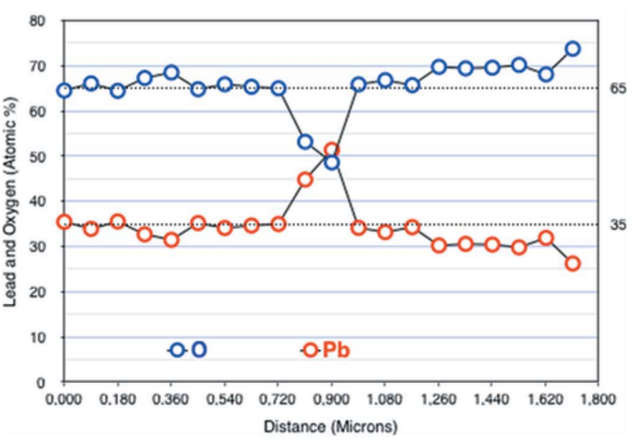

(c)

Figure 2

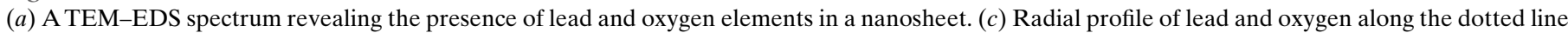

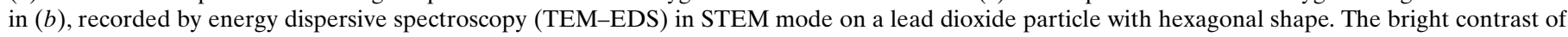

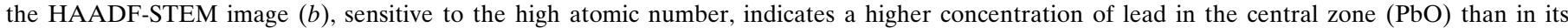
surroundings $\left(\mathrm{PbO}_{2}\right)$. 
richer in lead than the surrounding part. When compared with the surrounding part, this central zone of the nanosheet is distinguished by its bright contrast when the nanosheet is analysed in HAADF-STEM mode.

The TEM-EDS analysis and the HAADF-STEM observation are consistent and in agreement. In HAADF-STEM imaging, the brighter the contrast of a phase, the higher the atomic number of its constituent elements. It follows from these results that the $\mathrm{PbO}$ monoxide is located around the centre while the $\mathrm{PbO}_{2}$ dioxide is homogeneously distributed in the surrounding area.

We also note that there are cracks in the $\mathrm{PbO}$ central zone and/or at the $\mathrm{PbO} / \mathrm{PbO}_{2}$ interface. We will focus on this later when we discuss the oxidation of lead nanosheets after the evaporation of the liquid nitrogen and the possible impact on the mechanical properties of the lead oxide nanosheets.

\subsection{Crystal structure identification of $\gamma-\mathrm{PbO}_{2}$ : point and space groups and lattice parameters}

In TEM, the microdiffraction pattern corresponds to the intersection of the Ewald sphere with the zeroth and first reciprocal lattice layers recorded along $\langle u v w\rangle$, a zone axis of the phase that we are trying to identify. To these zeroth and first layers correspond the zero (ZOLZ) and the first (FOLZ) order Laue zones, respectively. The microdiffraction analysis is based on the net and ideal symmetries of the ZOLZ and of the whole pattern, $\mathrm{WP}=(\mathrm{ZOLZ}+\mathrm{FOLZ})$. The net symmetry concerns the position of reflections, while the ideal symmetry involves both the position and the intensity of these reflections. These symmetries, observed along specific zone axes, are connected to the crystal systems and to the point groups.

Fig. 3(a) shows a microdiffraction pattern recorded along the $\langle u v w\rangle$ zone axis of the $\gamma-\mathrm{PbO}_{2}$ particle. Taking into account both the position and the intensity of the reflections of this zone-axis pattern (ZAP), it is clear that the highest ideal symmetry for this phase is $\{(\mathbf{6 m m}), \underline{6 m m}\}$.

Considering this symmetry, the possible crystal system is the hexagonal one, with the only primitive $(P)$ Bravais unit cell (Morniroli \& Steeds, 1992). The possible point groups are then $6 \mathrm{~mm}$ or $6 / \mathrm{mmm}(6 / \mathrm{m} 2 / \mathrm{m} 2 / \mathrm{m}$, in its full notation; Morniroli \& Steeds, 1992; Redjaïmia \& Morniroli, 1994). Depending on this result, the $\langle u v w\rangle$ ZAP is indexed as [001] $=[0001]$.

The two potential candidates, $6 \mathrm{~mm}$ and $6 / \mathrm{m} 2 / \mathrm{m} 2 / \mathrm{m}$ point groups, can be distinguished by their corresponding ideal symmetries (2mm) and (m) for the ZOLZ when the nanosheets are observed along the $[100]=[2 \overline{1} 10]$ zone axis (Morniroli \& Steeds, 1992; Redjaïmia \& Morniroli, 1994). The latter is obtained when the hexagonal nanosheet is oriented edge on, as shown in Fig. 4(a).

Actually, the diffraction pattern, recorded under the conditions outlined previously, displays the (2mm) ideal symmetry [Fig. 4(b)]. This reveals (2)mm) ideal symmetry for the ZOLZ along the $[100]=[2110]$ zone axis, so we can discard the $6 \mathrm{~mm}$ point group in favour of $6 / \mathrm{m} 2 / \mathrm{m} 2 / \mathrm{m}$ (Morniroli \& Steeds, 1992; Redjaïmia \& Morniroli, 1994).
For the left $6 / m 2 / m 2 / m$ point group, four space groups satisfying such symmetry requirements are potential candidates, namely $P 6 / \mathrm{m} 2 / \mathrm{m} 2 / \mathrm{m}, P 6 / \mathrm{m} 2 / \mathrm{c} 2 / \mathrm{c}, P 6_{3} / \mathrm{m} 2 / \mathrm{m} 2 / \mathrm{c}$ and $P 6_{3} /$ $m 2 / c 2 / m$.

To distinguish between these four space groups, their simulated diffraction patterns along the [100] $=[2110]$ zone axis [Figs. 5(a) and 5(c)] are compared with the experimental

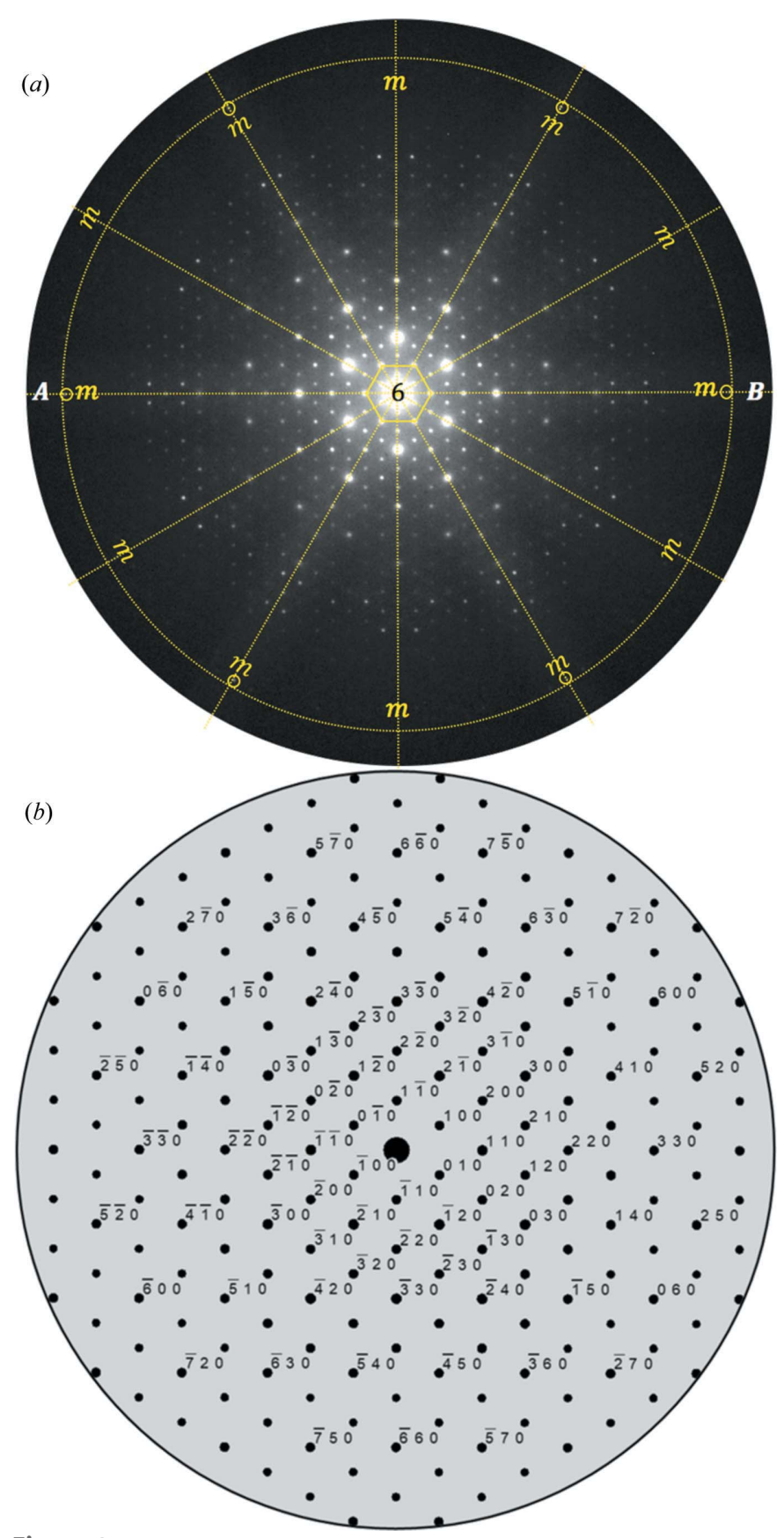

Figure 3

(a) Experimental and (b) the corresponding simulated (ZOLZ) electron microdiffraction patterns recorded along the $[001]=[0001]$ zone axis, showing $\{(\mathbf{6 m m}), \mathbf{6 m m}\}$ ideal symmetry for the whole pattern WP = $(\mathrm{ZOLZ}+\overline{\mathrm{FOLZ}})$. The small circles in $(a)$ mark the reflections belonging to the FOLZ indicated by the great circle. The radius of the circle is denoted by $R_{\mathrm{FOLZ}}$. 


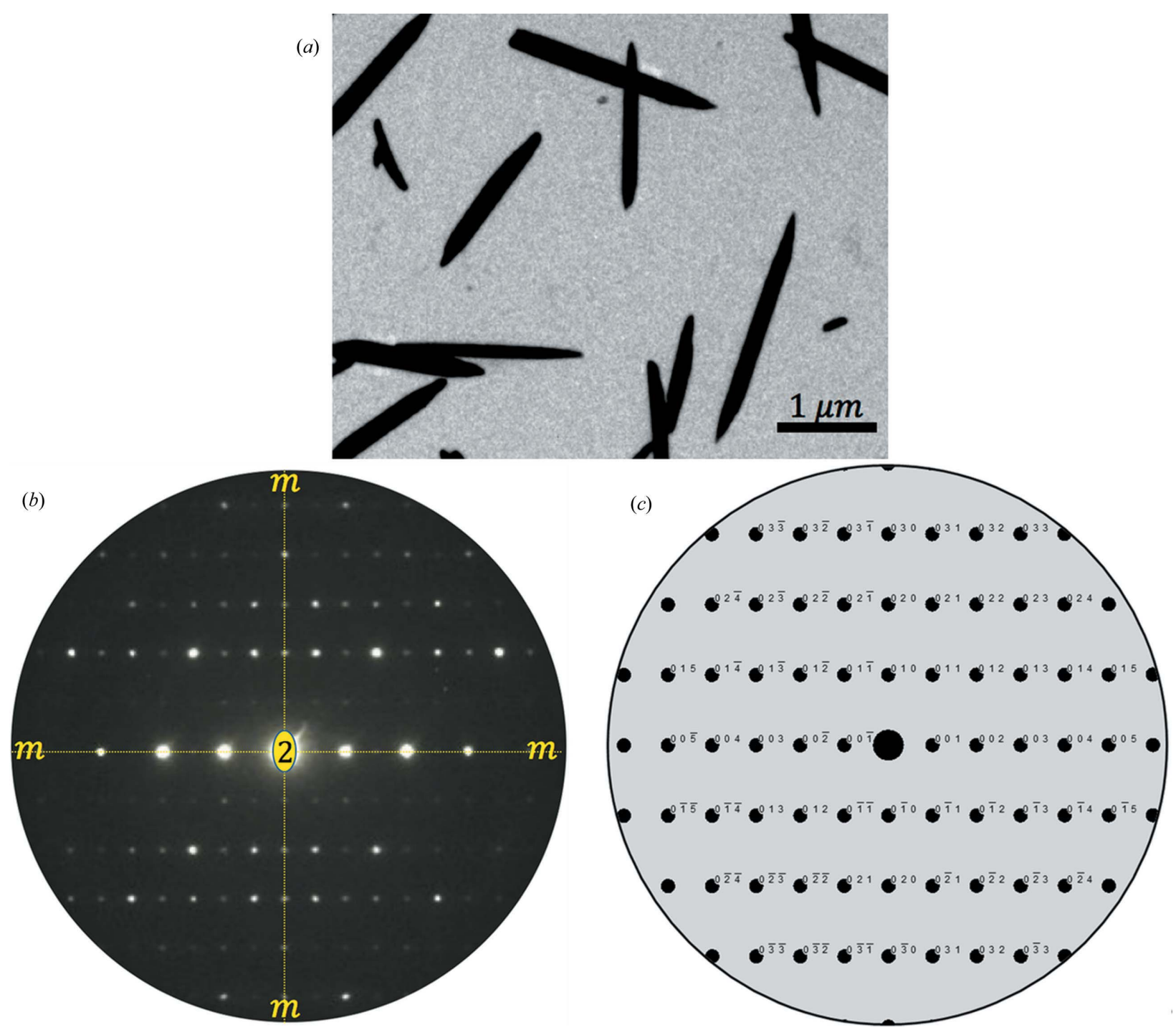

Figure 4

(a) Nanosheets oriented edge on in TEM with the corresponding $(b)$ experimental and $(c)$ simulated electron microdiffraction patterns recorded along the $[100]=[2 \overline{1} 10]$ zone axis. The experimental pattern shows a (2mm) ideal symmetry for the ZOLZ pattern.

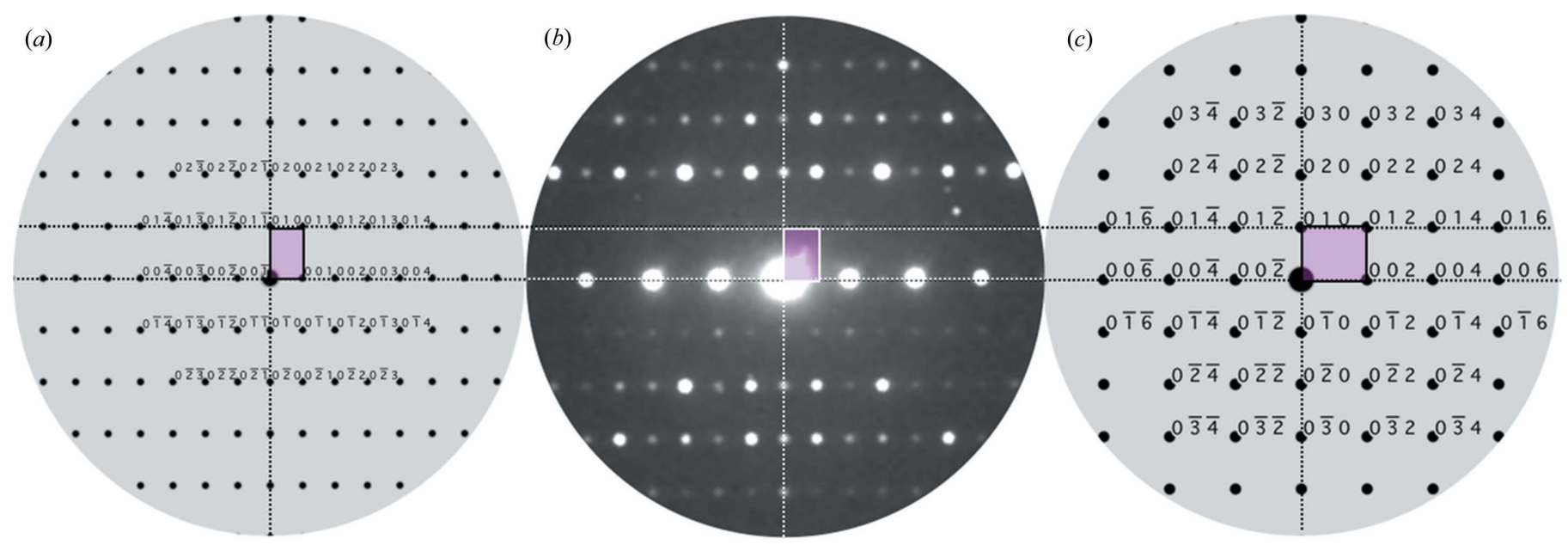

Figure 5

Comparison of the $(b)$ experimental electron diffraction pattern with the $(a),(c)$ simulated ones for the four space groups $(a) P 6 / m 2 / m 2 / m$ and $(c) P 6 / m 2 /$ $c 2 / c, P 6_{3} / \mathrm{m} 2 / \mathrm{m} 2 / c$ and $P 6_{3} / \mathrm{m} 2 / \mathrm{c} 2 / \mathrm{m}$. All the simulated patterns are recorded along the [100] $=[2 \overline{1} \overline{1} 0]$ zone axis. Only the diffraction pattern of $(a)$ the $P 6 /$ $\mathrm{m} 2 / \mathrm{m} 2 / \mathrm{m}$ space group matches with $(b)$ the experimental one. 
Table 1

Crystallographic and physical properties of the three polymorphic dioxides $\alpha-\mathrm{PbO}_{2}, \beta-\mathrm{PbO}_{2}$ and $\gamma-\mathrm{PbO}_{2}$, compared with natural lead, $\mathrm{Pb}$, and with the two polymorphic monoxides, $\alpha$ - $\mathrm{PbO}$ and $\beta$ - $\mathrm{PbO}$.

The hardness is expressed on the Mohs scale (ND = Not determined).

\begin{tabular}{|c|c|c|c|c|c|c|c|c|c|}
\hline \multirow{2}{*}{$\begin{array}{l}\text { Lead and lead monoxide } \\
\text { and dioxide allotriomorphs }\end{array}$} & \multicolumn{3}{|c|}{ Lattice parameters } & \multirow{2}{*}{$\begin{array}{l}\text { Crystal system and } \\
\text { space group }\end{array}$} & \multirow{2}{*}{$\begin{array}{l}V\left(\AA^{3}\right) \\
n \times\left(\mathrm{PbO}_{2}\right) \\
(\text { groups per unit cell) }\end{array}$} & \multirow{2}{*}{$\begin{array}{l}\text { Volumic mass } \\
\left(\mathrm{g} \mathrm{cm}^{-3}\right)\end{array}$} & \multirow{2}{*}{$\begin{array}{l}\text { Molar mass } \\
\left(\mathrm{g} \mathrm{mol}^{-1}\right)\end{array}$} & \multirow{2}{*}{$\begin{array}{l}\text { Molar volume } \\
\left(\mathrm{cm}^{3} \mathrm{~mol}^{-1}\right.\end{array}$} & \multirow{2}{*}{$\begin{array}{l}\text { Hardness } \\
\text { (Mohs scale) }\end{array}$} \\
\hline & $a(\AA)$ & $b(\AA)$ & $c(\AA)$ & & & & & & \\
\hline $\mathrm{Pb}$ (lead) & 4.951 & 4.951 & 4.951 & $\begin{array}{l}\text { Cubic } \\
F m \overline{3} m(225)\end{array}$ & $\begin{array}{l}121.36 \\
-\end{array}$ & 11.34 & 207.2 & 18.3 & 1.5 \\
\hline$\alpha$-PbO (litharge) & 3.97 & 3.97 & 5.02 & $\begin{array}{l}\text { Quadratic } \\
\text { P4/nmm (129) }\end{array}$ & $\begin{array}{l}79.20 \\
-\end{array}$ & 9.53 & 223.2 & 23.42 & 2 \\
\hline$\beta$ - $\mathrm{PbO}$ (massicot) & 5.489 & 5.755 & 5.891 & $\begin{array}{l}\text { Orthorhombic } \\
\text { Pbma (55) }\end{array}$ & $\begin{array}{l}186.09 \\
-\end{array}$ & 9.64 & 223.2 & 23.15 & 2 \\
\hline$\alpha-\mathrm{PbO}_{2}$ (scrutinyite) & 4.977 & 5.949 & 5.444 & $\begin{array}{l}\text { Orthorhombic } \\
\text { Pbcn (60) }\end{array}$ & $\begin{array}{l}161.19 \\
4\end{array}$ & 9.38 & 239.2 & 25.5 & 2 \\
\hline$\beta-\mathrm{PbO}_{2}$ (plattnerite) & 4.27 & 4.27 & 3.40 & $\begin{array}{l}\text { Quadratic } \\
\text { P4/nmm (129) }\end{array}$ & $\begin{array}{l}61.99 \\
2\end{array}$ & 9.5 & 239.2 & 25.2 & 5.5 \\
\hline$\gamma-\mathrm{PbO}_{2}($ novel $)$ & 9.12 & 9.12 & 12.59 & $\begin{array}{l}\text { Hexagonal } \\
\text { P6/mmm (191) }\end{array}$ & $\begin{array}{l}906.87 \\
36\end{array}$ & 15.76 & 239.2 & 15.2 & ND \\
\hline
\end{tabular}

one [Fig. 5(b)]. Only the simulated diffraction pattern of the $P 6 / m 2 / m 2 / m$ space group matches the experimental one.

The simulated diffraction patterns are based on the lattice parameters deduced from the experimental diffraction patterns recorded along the $[001]=[0001]$ and $[100]=[2 \overline{1} \overline{1} 0]$ zone axes.

The lattice parameters $a$ and $c$ are determined from SAED and microdiffraction patterns obtained along the same [001] = [0001] [Fig. 3(a)] and [100] $=[2 \overline{1} \overline{1} 0]$ [Fig. 4(b)] zone axes.

The $c$ lattice parameter was calculated starting from the following relation (Thomas \& Goringe, 1979):

$$
c=P_{[0001]}=2 \frac{(L \lambda)^{2}}{\lambda R_{\mathrm{FOLZ}}^{2}},
$$

where $P_{[0001]}$ is the row parameter, $\lambda=0.0035 \mathrm{~nm}$ is the electron wavelength for $V=120 \mathrm{kV}, L$ is the chamber length of the microscope working at $120 \mathrm{kV}$ and $R_{\mathrm{FOLZ}}$ is the radius of the FOLZ.

Since the intensity of the FOLZ is very low and difficult to access, the intensity profiles of the Laue zones (ZOLZ and FOLZ) have been plotted in different directions in order to assess the location of the FOLZ with the greatest possible accuracy and thus to determine the radius of the FOLZ as well as the row parameter and, finally, the $c$ lattice parameter.

In Fig. 6 is plotted the intensity profile of the $h h 0$ and $h h 1$ reflections belonging to the ZOLZ and FOLZ, respectively. The profile depicted in Fig. 6 is recorded along the $A B$ line of the diffraction pattern obtained along the $[001]=[0001]$ zone axis [Fig. 3(a)]. In Figs. 3(a) and 6, the reflection intensities in the ZOLZ decrease with increasing $R_{\text {FOLZ }}$ until the eighth reflection and increase significantly for those localized in the FOLZ. The profile indicates that $R_{\text {FOLZ }}$ extends to the tenth reflection, situated in the FOLZ, indexed as (10101). The results inferred from this procedure are $a=0.912 \mathrm{~nm}, c=$ $1.259 \mathrm{~nm}$ and $c / a=1.38$.
These values are in accordance with those determined from diffraction patterns recorded along other zone axes, especially $[100]=[2 \overline{1} \overline{1} 0]$.

These crystallographic investigations lead to the following results: the hexagonal-prismatic lead dioxide nanosheets crystallize in the hexagonal system with the $P$ Bravais lattice and belong to the $6 / \mathrm{mmm}$ holosymmetric point group and finally to the $P 6 / \mathrm{mmm}$ ( $P 6 / \mathrm{m} 2 / \mathrm{m} 2 / \mathrm{m}$, in its full notation) space group with the following lattice parameters: $a=0.912 \mathrm{~nm}$ and $c=1.259 \mathrm{~nm}$, with $c / a=1.38$. Note that the $c / a=1.38$ lattice parameter ratio is smaller than $c / a=(8 / 3)^{1 / 2}=1.63$, the characteristic value for hexagonal-close-packed metals.

To our knowledge, this hexagonal phase, labelled $\gamma-\mathrm{PbO}_{2}$, is the third polymorphic form, after the $\alpha-\mathrm{PbO}_{2}$ and $\beta-\mathrm{PbO}_{2}$ lead dioxides, the former being orthorhombic and the latter being tetragonal.

In Table 1, we have gathered the crystallographic and physical properties of the three polymorphic forms of $\mathrm{PbO}_{2}$ for comparison with natural lead and with $\alpha-\mathrm{PbO}$ and $\beta$-PbO, the two polymorphic forms of lead monoxide.

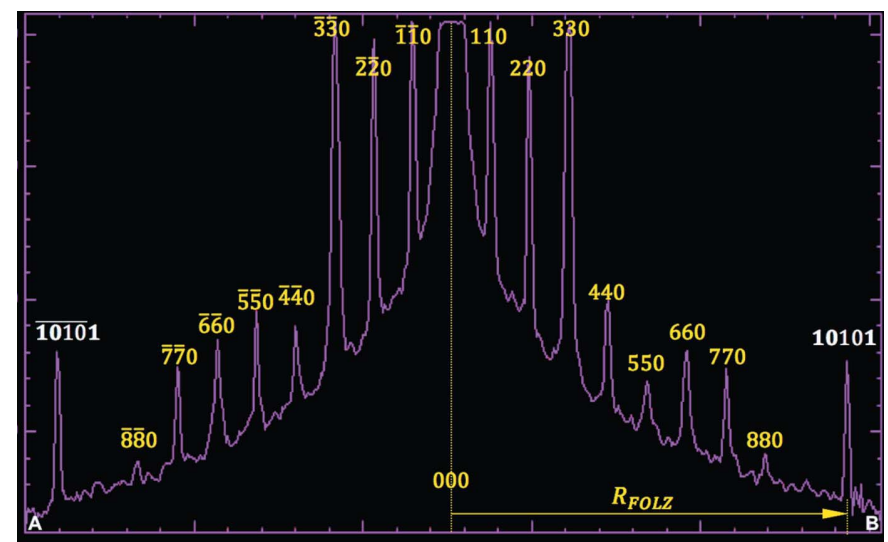

Figure 6

Intensity profile of $h h 0$ and $h h 1$ TEM reflections of the ZOLZ and FOLZ, respectively, along the line $A B$ [Fig. 3(a)] of the diffraction pattern recorded along the $[001]=[0001]$ zone axis. 


\subsection{Symmetry analysis and morphology, the equilibrium shape}

It is well established, through group theory, that the morphology of phases present in any medium (solid, liquid or gas) is of great importance in materials science. There have been significant efforts to understand both the equilibrium shape and the habit plane adopted by these phases in a particular medium (Cahn \& Kalonji, 1982; Redjaïmia et al., 1993). We will apply this group theory, based on the symmetry analysis, to explain the shape developed by the $\gamma-\mathrm{PbO}_{2}$ nanosheets.

A trace analysis derived from the electron diffraction pattern recorded along [001] $=[0001]$, superimposed on its corresponding TEM image [Fig. 7(a)], reveals that the $\gamma-\mathrm{PbO}_{2}$ particles adopt a hexagonal-prismatic shape. The lateral faces parallel to the $c$ axis, i.e. parallel to [001] $=[0001]$, and bounding the hexagonal prism are parallel to the following crystallographic planes:

$$
\{(1 \overline{1} \overline{2} 0),(2 \overline{1} 10),(1 \overline{2} \overline{1} 0),(\overline{1} \overline{1} 20),(\overline{2} 1 \overline{1} 0),(\overline{1} \overline{1} 20)\} \text {. }
$$

(Four digits are intentionally used in this paper for labelling planes and directions to emphasize their symmetry equivalence.)

It is well known that the shape of a crystallographic precipitate developing in a solid matrix is dictated by the point
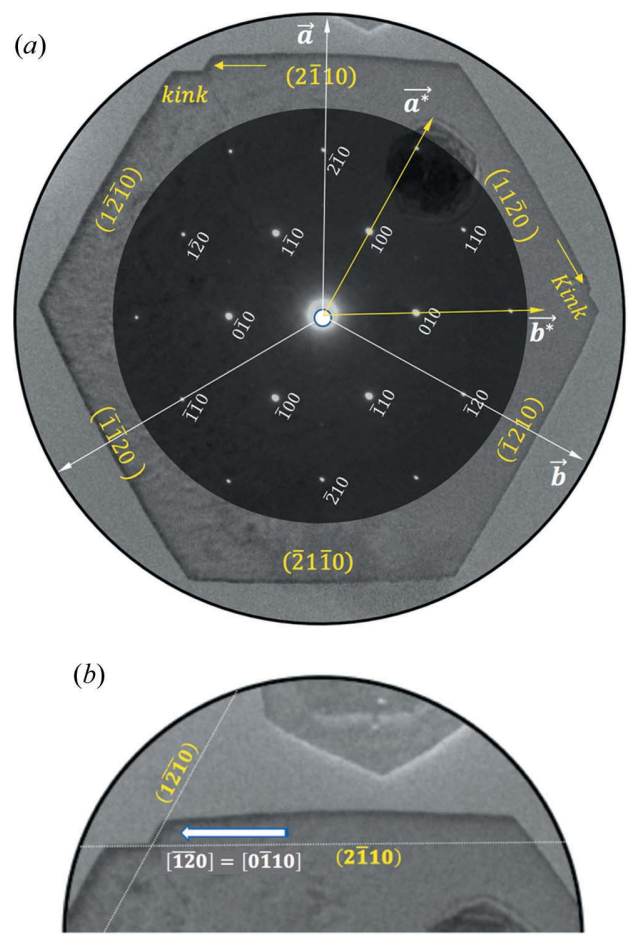

Figure 7

(a) Electron diffraction pattern recorded along the [001] $=[0001]$ zone axis and superimposed on the corresponding bright field TEM image of a lead dioxide particle. The triplets $(\mathbf{a}, \mathbf{b}, \mathbf{c})$ and $\left(\mathbf{a}^{*}, \mathbf{b}^{*}\right.$ and $\left.\mathbf{c}^{*}\right)$ are, for the hexagonal unit cell, the direct and reciprocal basis vectors, respectively. (b) Focus on one of the kinks shown at the top of Fig. 7(a). The characteristics of this kink are its terrace (2110) and its front (1210). group, the elements of which are common to the precipitate and to the matrix (Cahn \& Kalonji, 1982), while the shape of a phase occurring in an isotropic medium is imposed by the symmetry element of only its point group (Buerger, 1978).

On the basis of the electron diffraction pattern, we can assert that the $\gamma-\mathrm{PbO}_{2}$ lead dioxide particles, belonging to the 6/m2/m2/m point group, develop a hexagonal-prismatic shape (through the origin) (Phillips, 1971). This shape, corresponding to an absolute extremum (Cahn \& Kalonji, 1982), indicates that the development of the $\gamma-\mathrm{PbO}_{2}$ particles is energetically favourable, i.e. a stable lead dioxide. The results are in accordance with the symmetry analysis.

On close inspection of Fig. 7, the convexity of the faces of the lead dioxide nanosheet and the presence of kinks on lateral faces of the hexagonal prism of the lead dioxide particle are apparent.

In Fig. 7, the front of the kink is parallel to the (12 $\overline{1} 0)$ face and moves parallel to the $(11 \overline{2} 0)$ flat face in the $[\overline{1} \overline{2} 0]=[0 \overline{1} 10]$ direction.

\subsection{Growth mechanism of the lead dioxide particle}

The $\gamma-\mathrm{PbO}_{2}$ phase is formed by oxidation of lead nanosheets, in contact with air, after evaporation of liquid nitrogen. It is therefore important to understand which mechanism is involved in the formation of lead nanosheets in liquid nitrogen. In this section, we will try to develop this mechanism.

The hexagonal-prismatic shape and the particular dimensions of the particles lead us to question the formation mechanism of these particles of lead nanosheet in liquid nitrogen, which can be considered an isotropic medium. To answer this question, special attention to the diffraction pattern and its corresponding TEM image (Fig. 7) is required. From these figures, two characteristics can be drawn:

(1) The particle lateral faces are convex or at least flat but never concave [Fig. 7(a)].

(2) Most of the particle faces are decorated by kinks. The latter are characterized by their terraces and their front [Figs. $7(b)$ and $8(b)$ ].

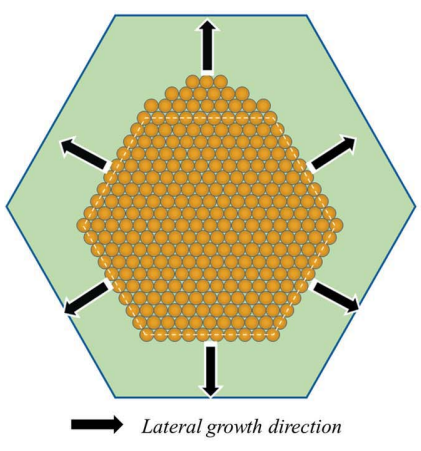

(a)

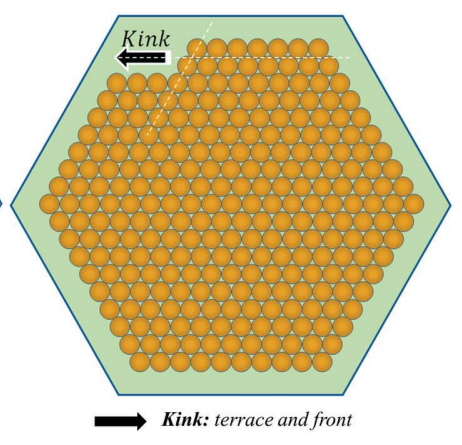

(b)
Figure 8

(a) Schematic representation of the radial growth of the lead nanosheet. At the mesoscopic level, the nanosheets are convex and can be decorated by kinks (Fig. 7). On one of the prism faces in (a), the convexity is exaggerated to emphasize the radial expansion of the nanosheet. (b) Presence of a kink on one lateral face. 
These experimental observations remind us of the modes by which thin films grow epitaxially on a crystal surface or at an interface, especially those of Frank \& Van der Merwe (1949), Stranski \& Krastanow (1939) and Volmer \& Weber (1926), or those describing the development of precipitates in a metallic matrix, known as the ledge mechanism.

In our case, the terrace and the front of a kink are parallel to the lateral face where it is located and to the following adjacent face, respectively [Fig. 7(a)].

The hexagonal lead oxide particles are very thin (around $10 \mathrm{~nm})$ and well developed radially $(1 \mu \mathrm{m})$. The fact that the particles are convex (or at least flat) indicates that they nucleate and grow radially, as indicated in Fig. 8(a).

The presence of kinks on the lateral faces strongly suggests that the growth mechanism is akin to the well known ledge mechanism where the matrix is replaced by an isotropic medium (Porter et al., 2009). The scenario of lateral growth by the ledge mechanism is illustrated by the schematic representation in Fig. $8(b)$.

In the case of the ledge mechanism, the growth of the precipitate is dictated by the coherency of the interfaces between the precipitate and the matrix where the growth takes place. The development of the precipitate is more important for the incoherent interfaces than for the coherent ones. The lateral growth of the precipitate is faster than its thickening (Porter et al., 2009).

In our case, we have the same scenario except that the matrix is replaced by the liquid nitrogen. Consequently, the interface can no longer be considered to be coherent.

We think that, in liquid nitrogen, the development of the lead nanosheet crystal is attributable to the compactness of its atomic planes. The rougher the plane, i.e. the lower the density, the faster the development perpendicular to that plane.

Placed in the $\gamma-\mathrm{PbO}_{2}$ reference, the lead nanosheet will develop perpendicularly to the $\{11 \overline{2} 0\}$ planes, less dense than (0001), as indicated in Fig. 8. In other words, the lateral growth velocity of the lead nanosheet is more important than its thickening. The thickening is inhibited, leading to lead nanosheets.

The compactness, a primary physical property, appears to control the morphology of the lead nanosheet in liquid nitrogen.

\subsection{Oxidation of the lead nanosheet, formation of the $\gamma-\mathrm{PbO}_{2}$ nanosheet}

In the HAADF-STEM micrographs [Figs. $9(a)$ and $9(b)$ ], it is noted that the bright central nanosheet zone is richer in lead $(\mathrm{PbO})$ than the surrounding part $\left(\mathrm{PbO}_{2}\right)$. This observation suggests that, after the evaporation of the liquid nitrogen, the oxygen would diffuse radially from the periphery to the centre of the particle, unlike the radial growth of the lead particle.

Let us recall that the radial growth direction is perpendicular to the $\{11 \overline{2} 0\}$ plane family, less dense than the (0001) plane.

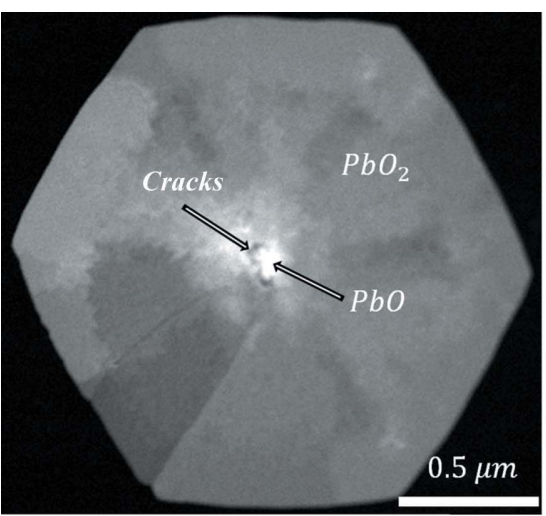

(a)

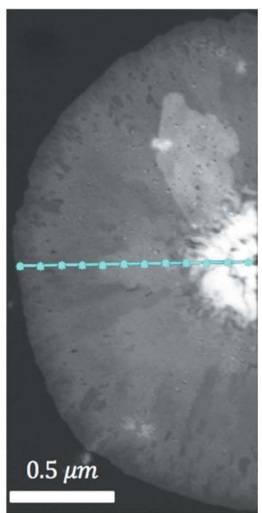

(b)
Figure 9

HAADF-STEM micrographs. The bright contrast is sensitive to the high atomic number and indicates qualitatively the presence of more lead in the central zone than in the outer. This result is quantitatively corroborated by the radial distribution of lead and oxygen along the dotted line $(b)$ recorded by energy dispersive spectroscopy (TEM-EDS) on a lead dioxide particle: the $\mathrm{PbO}$ monoxide is located around the centre while the $\mathrm{PbO}_{2}$ dioxide is homogeneously distributed in the surrounding area. However, there are cracks around the $\mathrm{PbO}$ central zone at the $\mathrm{PbO} /$ $\mathrm{PbO}_{2}$ interface $(a)$.

The formation of $\mathrm{PbO}$ instead of $\mathrm{PbO}_{2}$ in the central zone of certain particles can probably be attributed to a partial oxidation of the lead nanosheet due to an oxygen deficiency during its radial diffusion.

From Table 1, it can be seen that the volume change between $\mathrm{PbO}$ and $\mathrm{PbO}_{2}$, more than $50 \%$, would induce a concentration of strong stresses, leading to cracking of the particle at its centre or at the $(\mathrm{PbO}) /\left(\mathrm{PbO}_{2}\right)$ interface, as shown in Fig. 9(a).

\section{Conclusion and discussion}

Through this study, we have shown that the synthesis of the novel $\gamma-\mathrm{PbO}_{2}$ nanosheets originates from spark discharge, in the liquid nitrogen, followed by oxidation in air. This new polymorphic dioxide crystallizes in the hexagonal system $(a=$ $0.912 \mathrm{~nm}$ and $c=1.259 \mathrm{~nm}$ ) and belongs to the $6 \mathrm{~m} 2 / \mathrm{m} 2 \mathrm{~m}$ holosymmetric point group and to the $P 6 / \mathrm{m} 2 / \mathrm{m} 2 / \mathrm{m}$ space group.

On the basis of group theory (symmetry analysis), the $\gamma-\mathrm{PbO}_{2}$ polymorph develops a hexagonal-prismatic shape inherited from the lead nanosheets formed in liquid nitrogen. From the energetic point of view, this shape corresponds to an absolute extremum, which results in a stable state of the nanosheet.

A mechanism similar to that of the ledge mechanism explaining the formation of thin plates in a metallic matrix has been adapted and proposed. In contrast to the plane coherency in the ledge mechanism, the plane compactness seems to play an important role in the development of the lead nanosheets in liquid nitrogen, before their oxidation in air.

As far as we are aware, this is the first time that a such lead dioxide, designated $\gamma-\mathrm{PbO}_{2}$, has been synthesized, its crystal- 
lographic structure determined, its morphology explained and a mechanism for its development proposed.

It is legitimate to question the crystal structure of the particle formed in the liquid nitrogen. However, the answer remains technically difficult if not impossible to find. Indeed, the new lead dioxide, $\gamma-\mathrm{PbO}_{2}$, could originate from either a lead whose structure is face-centred cubic, like that of the natural lead, or another hypothetical 'allotropic' variety of the lead with hexagonal structure, described by an $\ldots A B C A B C \ldots$ or an $\ldots A B A B A B \ldots$ close-packed stacking, respectively.

Following the evaporation of the liquid nitrogen, two cases can naturally be envisaged for the genesis of the lead dioxide $\gamma-\mathrm{PbO}_{2}$ :

(1) The native face-centred cubic lead oxidizes and gives rise to the new polymorphic hexagonal phase, $\gamma-\mathrm{PbO}_{2}$. A phase transformation, face-centred cubic to hexagonal structure, takes place,

(2) The 'hypothetical' native hexagonal lead oxidizes and gives rise to the new polymorphic hexagonal phase, $\gamma-\mathrm{PbO}_{2}$, by incorporation of oxygen in lead.

It is recalled that $\alpha-\mathrm{PbO}_{2}$, a rare mineral named scrutinyite, was first identified in nature in 1988 and was only synthesized in 1941. The $\beta-\mathrm{PbO}_{2}$ phase, a more common mineral named plattnerite, was first identified around 1845 and later synthetically produced.

Analysis of Table 1 shows that, in the case of lead dioxide, the calculated volume change in expansion from the orthorhombic form or from the quadratic form to the hexagonal one is very high, more than $50 \%$.

However, it is well known that ceramics are very brittle and cannot sustain more than a $0.1 \%$ change in volume. This would explain why the $\gamma-\mathrm{PbO}_{2}$ polymorph was only synthesized by the nanosecond-pulsed spark discharge between two lead electrodes in liquid nitrogen followed by oxidation in air and never by the classical polymorphic transformation, involving temperature and/or pressure variations.

Finally, to avoid high volume changes in expansion and take advantage of the spark discharge, metallic elements or oxides of different elements (magnesium, zirconium, yttrium etc.) could be added to stabilize the $\mathrm{PbO}_{2}$ in another polymorphic form with desired and targeted properties.

\section{References}

Aramini, J., Yazawa, T., Yuan, T., Zhang, M. \& Vogel, H. (1996). JBIC, 1, 39-48.

Belmonte, T., Hamdan, A., Kosior, F., Noël, C. \& Henrion, G. (2014). J. Phys. D Appl. Phys. 47, 224016.

Buerger, M. J. (1978). Elementary Crystallography. Cambridge: Massachussetts Institute of Technology Press.

Cahn, J. W. \& Kalonji, G. (1982). Proceedings of the International Conference on Solid-Solid Phase Transformations, edited by H. I. Aaronson, R. F. Sekereka, D. E. Laughlin \& C. M. Waymann, pp. 3 14. Warrendale: TMS-AIME.

Charinpanitkul, T., Soottitantawat, A., Tonanon, N. \& Tanthapanichakoon, W. (2009). Mater. Chem. Phys. 116, 125-128.

Frank, F. C. \& Van der Merwe, J. (1949). Proc. R. Soc. London Ser. A, 198, 216-225.

Hamdan, A., Kabbara, H., Noel, C., Ghanbaja, J., Redjaimia, A. \& Belmonte, T. (2018). Particuology, 40, 152-159.

Hamdan, A., Noel, C., Kosior, F., Henrion, G. \& Belmonte, T. (2013). J. Appl. Phys. 113, 043301.

Haviland, D., Liu, Y. \& Goldman, A. (1989). Phys. Rev. Lett. 62, 2180-2183.

Morniroli, J. P. \& Steeds, J. W. (1992). Ultramicroscopy, 45, 219-239.

Phillips, F. C. (1971). An Introduction to Crystallography, 4th ed. Singapore: Longman.

Porter, D. A., Easterling, K. E. \& Sherif, M. Y. (2009). Phase Transformations in Metals and Alloys, 3rd ed. Boca Raton: CRC Press.

Redjaïmia, A. \& Morniroli, J.-P. (1994). Ultramicroscopy, 53, 305-317. Redjaïmia, A., Ruterana, P., Metauer, G. \& Gantois, M. (1993). Philos. Mag. A, 67, 1277-1286.

Sano, N., Kikuchi, T., Wang, H., Chhowalla, M. \& Amaratunga, G. A. (2004). Carbon, 42, 95-99.

Stranski, I. N. \& Krastanow, L. (1939). Monatsh. Chem. 71, 351-364. Strongin, M., Thompson, R., Kammerer, O. \& Crow, J. (1970). Phys. Rev. B, 1, 1078-1091.

Thomas, G. \& Goringe, M. J. (1979). Transmission Electron Microscopy of Materials. New York, Chichester, Brisbane, Toronto: John Wiley and Sons.

Volmer, M. \& Weber, A. (1926). Z. Phys. Chem. 119, 277-301. 